\title{
Imaginary Chemical Potential Approach for the Pseudo-Critical Line in the QCD Phase Diagram with Clover-Improved Wilson Fermions
}

\author{
Keitaro Nagata ${ }^{1,2, *}$ and Atsushi Nakamura ${ }^{2, \dagger}$ \\ ${ }^{1}$ Department of Physics, The University of Tokyo, Bunkyo-ku, Tokyo 113-0033 JAPAN \\ ${ }^{2}$ Research Institute for Information Science and Education, \\ Hiroshima University, Higashi-Hiroshima 739-8527 JAPAN
}

(Dated: November 12, 2018)

\begin{abstract}
The QCD phase diagram is studied in the lattice QCD simulation with the imaginary chemical potential approach. We employ a clover-improved Wilson fermion action of two-flavors and a renormalization-group improved gauge action, and perform the simulation at an intermediate quark mass on a $8^{3} \times 4$ lattice. The QCD phase diagram in the imaginary chemical potential $\mu_{I}$ region is investigated by performing the simulation for more than 150 points on the $\left(\beta, \mu_{I}\right)$ plane. We find that the Roberge-Weiss phase transition at $\mu_{I} / T=\pi / 3$ is first order and its endpoint is second order, which are identified by the phase of the Polyakov loop. We determine the pseudo-critical line from the susceptibility of the Polyakov loop modulus. We find a clear deviation from a linear dependence of the pseudo-critical line on $\mu_{I}^{2}$.
\end{abstract}

PACS numbers: 25.75.Nq, 12.38.Mh, 21.65.Qr, 12.38.Gc

\section{INTRODUCTION}

The QCD phase diagram, which includes states of matter formed in terms of the strong interaction, has been of prime interest in recent physics covering particle physics, hadron/nuclear physics and astrophysics. Because QCD is non-perturbative in most regions of the QCD phase diagram, one is forced to use the lattice QCD in order to obtain a quantitative understanding. The lattice QCD is expected to provide reliable information on the phase structure based on QCD. Indeed, recently, there have been many active quantitative investigations about the finite temperature QCD [1, 2].

On the other hand, simulations of systems with nonzero quark chemical potential $\mu$ have been a long challenge for the lattice QCD because of the notorious sign problem. In the lattice QCD, a fermionic determinant $\operatorname{det} \Delta(\mu)$ is used as a probability in a Monte Carlo method. The introduction of non-zero $\mu$ makes $\operatorname{det} \Delta(\mu)$ complex, and therefore leads to the breakdown of the stochastic part of the lattice QCD, see Ref. [3].

Despite of the severe sign problem, several approaches have been proposed to study the QCD with

nagata@rcnp.osaka-u.ac.jp

†nakamura@ riise.hiroshima-u.ac.jp nonzero $\mu$, see e.g. [3, 4]. One idea is to avoid the sign problem by performing simulations in systems with an imaginary chemical potential. A partition function and its free-energy are analytic within one phase even if chemical potential is extended to complex, which is true until the occurance of a phase transition. This validates the imaginary chemical potential approach for the study of the QCD phase diagram.

Fermion determinants satisfy a well-known relation

$$
\Delta(\mu)^{\dagger}=\gamma_{5} \Delta\left(-\mu^{*}\right) \gamma_{5},
$$

which holds for a complex chemical potential: $\mu=$ $\mu_{R}+i \mu_{I}\left(\mu_{R}, \mu_{I} \in \mathbb{R}\right)$. This implies that $\operatorname{det} \Delta(\mu)$ is complex for a real chemical potential $\mu=\mu_{R}$, which causes the sign problem. On the other hand, one can easily prove $\operatorname{det} \Delta(\mu)$ is real for a pure imaginary chemical potential $\mu=i \mu_{I}$. The sign problem does not occur in this case, and Monte Carlo methods are available. The imaginary chemical potential approach provides an insight into the QCD phase diagram through the analytic continuation. In addition, data obtained in such a simulation can be used for the matching of phenomenological models such as Polyakov loop extended Nambu-Jona-Lasinio(PNJL) models with the lattice QCD [5, 6].

The imaginary chemical potential approach has been studied by using staggered fermions with two flavor 7 9], three flavor [10], four flavor [11-14] in 2-color 


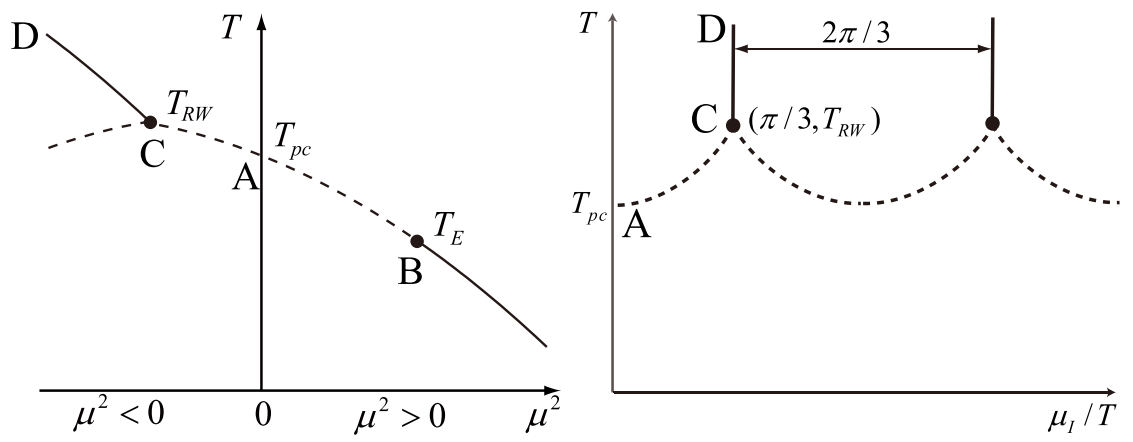

FIG. 1: Schematic figures for the $N_{f}=2$ QCD phase diagram in the $\left(\mu^{2}, T\right)$ plane (left) and $\left(\mu_{I} / T, T\right)$ plane (right). A : Pseudo-critical point at $\mu=0$. B : Critical endpoint. C : RobergeWeiss endpoint. AB : Pseudo-critical line. AC : Extension of the line AB into the imaginary chemical potential plane. CD : Roberge-Weiss phase transition line $\mu_{I} / T=\pi / 3$. In the right panel, larger $\mu_{I} / T$ region of the phase diagram is obtained from the RW periodicity.

QCD and finite isospin QCD [15, 16], by using Wilson fermions with two flavor [17].

Staggered fermions of the standard type might have suffered from two problems. First, it needs a fourthroot trick for one flavor [30]. Second, it does not show a scaling behavior expected from three-dimensional $\mathrm{O}(4)$ spin models for the finite temperature transition [18], although the possibility of the first oder phase transition for two degenerate flavors was studied in Ref. [19]. Wilson fermions are free from the fourth root tricks and show the correct scaling behavior. On the other hand, Wilson fermions suffer from an explicit breaking of chiral symmetry. However, one can define a subtracted chiral condensate, which satisfy a correct scaling behavior. Although Wilson fermions require more computational time than that required in staggered fermions, simulations with Wilson fermions are now possible even on the physical quark masses at zero density.

In finite temperature simulations with the combination of the plaquette gauge action and the standard Wilson quark action at $N_{t}=4$, the transition is smooth crossover at small and large quark mass and rapid crossover at intermediate quark mass [20], which is different from what is expected the transition becomes sharp for light and heavy quark masses. This unexpected behavior is removed by improving the gauge action [18, 21]. The improvement of the gauge action is essential in removing lattice artifacts at finite lattice spacings.
Thus, the study of the Wilson fermions with improved terms is complementary and useful to confirm results obtained in other actions [7-13, 17] and to establish a better understanding of the QCD phase diagram. In this paper, we study the two-flavor QCD phase diagram at an intermediate quark mass by using the imaginary chemical potential approach. This is the first employment of the two-flavor Wilson fermion with a clover term and the renormalization-group(RG) improved gauge action to the imaginary chemical potential approach.

This paper is organized as follows. In the next section, we briefly review properties and issues of the imaginary chemical potential region of the phase diagram. The setup for the simulation is also explained here. The numerical results are shown in Sec. IIII. We investigate the deconfinement transition and RobergeWeiss endpoint in Sec. IIIA and Roberge-Weiss phase transition line in Sec. IIIB We determine the pseudocritical line in Sec. IIIC, The final section is devoted to a summary.

\section{FRAMEWORK}

\section{A. Phase diagram with imaginary chemical potential}

We begin with a brief overview of the QCD phase diagram and of issues in question.

First we show in the left panel of Fig. 1 1 an expected phase diagram in $\left(\mu^{2}, T\right)$ plane containing both the real 
$\left(\mu^{2} \geq 0, \mu=\mu_{R}\right)$ and imaginary $\left(\mu^{2} \leq 0, \mu=\right.$ $\left.i \mu_{I}\right)$ regions. Even if $\mu^{2} \leq 0$, it is expected quarkgluon-plasma(QGP) and hadronic phases exist at high and low temperatures, respectively. The two phases are separated by the deconfinement crossover transition line, which is an extension from the $\mu^{2} \geq 0$ region. These are consequences of an analyticity of the grand partition function. The absolute value of the Polyakov loop is often employed to identify confinement/deconfinement phase, although it is not a real order parameter because of the crossover nature of the transition.

Two characteristics of the $\mu^{2} \leq 0$ region are so-called Roberge-Weiss(RW) phase transition and Roberge-Weiss(RW) periodicity [22]. The QCD grand partition function has a periodicity with a period $2 \pi / N_{c}$ as

$$
Z\left(\frac{\mu_{I}}{T}\right)=Z\left(\frac{\mu_{I}}{T}+\frac{2 \pi k}{N_{c}}\right),
$$

where $k$ is an integer. Furthermore, Roberge and Weiss showed from a perturbative analysis the existence of a first-order phase transition on the line $\mu_{I} / T=\pi / N_{c}$, and from a strong coupling analysis the absence of such a transition at low temperatures. These features hold for $\mathrm{SU}\left(N_{c}\right)$ gauge theories. Hereafter we consider $N_{c}=3$. The RW phase transition relates to the $Z(3)$ symmetry and an order parameter identifying this phase transition is the phase or imaginary part of the Polyakov loop. Because the RW phase transition occurs at high temperatures but does not at low temperatures, it may have an endpoint at a temperature $T_{R W}$ on the line $\mu_{I} / T=\pi / 3$. These features are well manifested in the $\left(\mu_{I} / T, T\right)$-phase diagram, see the right panel of Fig.1]

Although the phase diagrams in Fig. 1 are naively expected, several points remain as issues which should be discussed further. Figure 1 is drawn according to the two points :

- The RW phase transition exists at high temperatures and has an endpoint.

- The extension of the crossover line exists in the $\mu^{2} \leq 0$ region.

The second point needs an assumption that a pseudocritical line can be defined for a crossover. Once the
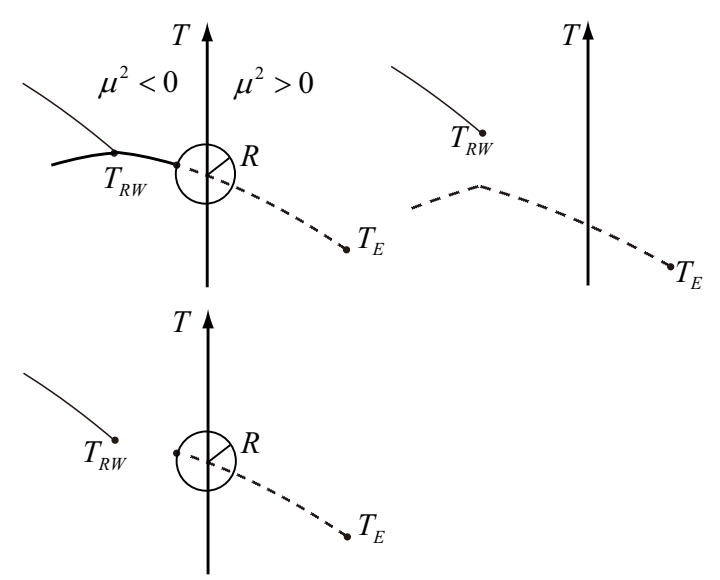

FIG. 2: Counter examples against the naive expectation. $R$ is a convergence radius of the pseudo-critical line determined in the $\mu^{2} \leq 0$ region.

pseudo-critical line is defined, the second point is ensured by the identity theorem.

Based on the above two points, one can consider several counter examples, against the naive expectation Fig. 1, see Fig. 2] In order to reveal the phase structure without assumptions on imagination, we need to know

- the location and order of the RW endpoint

- the location of phase boundary of the deconfinement transition

- the way with which the RW and deconfinement transition lines are connected

Indeed, the quark-mass dependence of the RW endpoint was found in Ref. [8, 10]. D'Elia et al discussed [8] a possibility that other first-order phase transition lines depart from the RW endpoint, which corresponds to the left top panel in Fig 2

The above questions are important for two reasons. First, they are relevant with the definition range of the pseudo-critical line determined by the imaginary chemical potential approach, which also relates to the applicable range of the line in the $\mu^{2} \geq 0$ region. For instance, if the pseudo-critical line has an endpoint near $T_{p c}$ at $\mu=0$ [31], then a convergence radius $R$ of the pseudo-critical line obtained from the imaginary chemical potential approach is given by a distance between the endpoint and $T_{p c}$. The pseudo-critical line obtained 
can be applied to a domain with the convergence radius.

Second, it is speculated from the Lee-Yang theorem [23, 24] that a phase transition in the $\mu^{2} \leq 0$ region is relevant to one in the $\mu^{2} \geq 0$ region, although the distribution of the Lee-Yang zeros of the QCD grand partition function has not been well understood. Assuming the Lee-Yang zeros of the QCD are distributed on a line in the complex fugacity plane, it is possible that the RW phase transition line and its endpoint in the $\mu^{2} \leq 0$ region reflect the first order phase transition line and critical endpoint in the $\mu^{2} \geq 0$ region of the
QCD phase diagram.

\section{B. Formulation and setup}

We employ the RG-improved gauge action [25]

$S_{g}=\frac{\beta}{6}\left[c_{0} \sum_{x, \mu<\nu} W_{\mu \nu}^{1 \times 1}(x)+c_{1} \sum_{x, \mu, \nu} W_{\mu \nu}^{1 \times 2}(x)\right]$

with $c_{1}=-0.331$ and $c_{0}=1-8 c_{1}$, and the cloverimproved Wilson fermion action with the quark matrix

$$
\begin{aligned}
\Delta(x, y)=\delta_{x, x^{\prime}} & -\kappa \sum_{i=1}^{3}\left[\left(1-\gamma_{i}\right) U_{i}(x) \delta_{x^{\prime}, x+\hat{i}}+\left(1+\gamma_{i}\right) U_{i}^{\dagger}\left(x^{\prime}\right) \delta_{x^{\prime}, x-\hat{i}}\right] \\
& -\kappa\left[e^{+\mu}\left(1-\gamma_{4}\right) U_{4}(x) \delta_{x^{\prime}, x+\hat{4}}+e^{-\mu}\left(1+\gamma_{4}\right) U_{4}^{\dagger}\left(x^{\prime}\right) \delta_{x^{\prime}, x-\hat{4}}\right] \\
& -\kappa C_{S W} \delta_{x, x^{\prime}} \sum_{\mu \leq \nu} \sigma_{\mu \nu} F_{\mu \nu} .
\end{aligned}
$$

Here $\mu$ is the quark chemical potential in lattice unit, which is introduced to the temporal part of link variables.

In order to scan the phase diagram, simulations were done for more than 150 points on the $\left(\mu_{I}, \beta\right)$ plane in the domain $0 \leq \mu_{I} \leq 0.28800$ and $1.79 \leq \beta \leq 2.0$. Note that the RW phase transition line in the present setup is given by $\mu_{I}=\pi / 12 \sim 0.2618$. All the simulations were performed on a $N_{s}^{3} \times N_{t}=8^{3} \times 4$ lattice. The value of the hopping parameter $\kappa$ were determined for each value of $\beta$ according to a line of the constant physics with $m_{P S} / m_{V}=0.8$ obtained in Ref. [26]. The coefficient of the clover term $C_{S W}$ was determined by using a result obtained in the one-loop perturbation theory [27] : $C_{S W}=\left(1-0.8412 \beta^{-1}\right)^{-3 / 4}$.

The hybrid Monte Carlo algorithm were employed to generate gauge configurations. The setup for the molecular dynamics was as follows: a step size $\delta \tau=$ 0.02 , number of the molecular dynamics $N_{\tau}=50$ and length $N_{\tau} \delta \tau=1$. The acceptance ratio for this setup was more than $90 \%$. We generated 11, 000 trajectories for most parameter sets, and 16, 000 trajectories for some parameter sets near the deconfinement tran- sition at $\mu_{I}=0$. For all the ensemble, the first 5,000 trajectories were removed as thermalization. The plaquette $P$, Polyakov loop $L e^{i \phi}$ and their susceptibilities were measured for each trajectory, where $L$ and $\phi$ are the modulus and phase of the Polyakov loop.

The Polyakov loop operator is as usual defined by

$$
P_{o l}=\frac{1}{N_{V} N_{c}} \sum_{x} \operatorname{tr} \prod_{t=1}^{N_{t}} U_{4}(\vec{x}, t)
$$

where $N_{V}=N_{s}^{3}$. The modulus and phase are, after the ensemble average, defined by $\left\langle P_{o l}\right\rangle=L e^{i \phi}$. Their susceptibilities are also defined by

$$
\begin{aligned}
& \chi_{L}=N_{V}\langle(L-\langle L\rangle)\rangle^{2}, \\
& \chi_{\phi}=N_{V}\langle(\phi-\langle\phi\rangle)\rangle^{2} .
\end{aligned}
$$

Distributions of the Polyakov loop in the complex plane are shown in Fig. 3. As the figures clearly show, the phase structure can be identified by considering the $\beta$ and $\mu_{I}$ dependence of the Polyakov loop. 

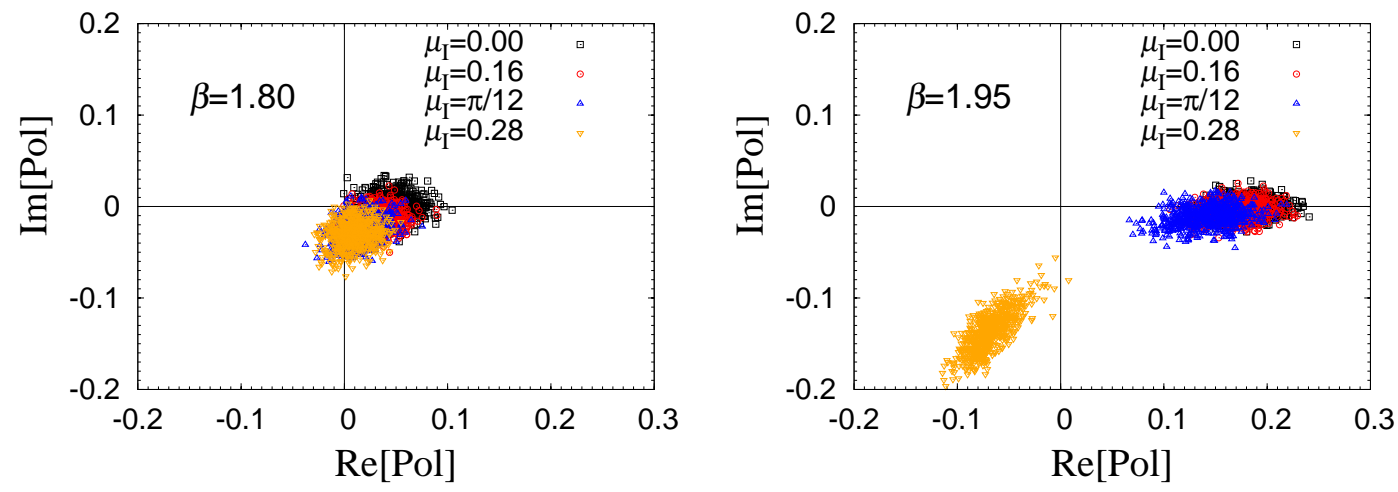

FIG. 3: Scatter plots of the Polyakov loop. Left : $\beta=1.80$ (low temperature (below $T_{p c}$ )). Right : $\beta=1.95$ (high temperature (above $T_{R W}$ )).
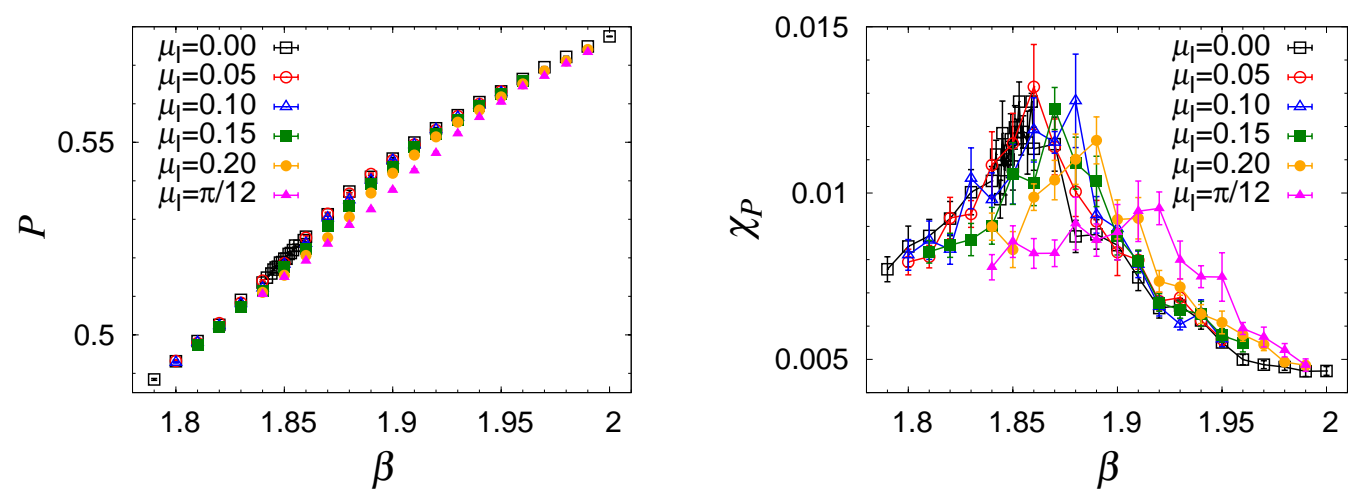

FIG. 4: The $\beta$-dependence of the plaquette $P$ (left) and its susceptibility $\chi_{P}$ (right) for various $\mu_{I}$.

\section{NUMERICAL RESULTS}

\section{A. Deconfinement transition and RW endpoint}

First, we investigate the deconfinement transition and RW endpoint, by considering the $\beta$-dependence of the observables.

The plaquette $P$ and its susceptibility $\chi_{P}$ are shown in Fig. 4. $P$ is a smooth increasing function of $\beta$ for all $\mu_{I}$. The effect of $\mu_{I}$ suppresses $P$ for intermediate $\beta$, while does not change it for small and large $\beta$. However, the effect is up to a few percent. $\chi_{P}$ has a broad peak, and the peak position moves toward larger $\beta$ with increasing $\mu_{I}$.

The Polyakov loop modulus $L$ and its susceptibility $\chi_{L}$ are shown in Fig. 5] $L$ increases slowly for small $\beta$. At a certain value of $\beta$, the slope of $L$ becomes large, and $\chi_{L}$ has a broad peak. These behaviors suggest the possibility that the system undergoes the crossover transition with increasing $\beta$ or temperature. The peak position tends to move towards a larger $\beta$ with increasing $\mu_{I}$, similar to the behavior of $\chi_{P}$. This behavior confirms that pseudo-critical temperatures become higher with the increase of $\mu_{I}$ until $\mu_{I}=\pi / 12$.

It should be noted that the crossover behavior is observed for all $\mu_{I}$, even on the line $\mu_{I}=\pi / 12$. Hence, the pseudo-critical line starts from $\mu_{I}=0$ and reaches $\mu_{I}=\pi / 12$.

Also note that the peak of $\chi_{P}$ and $\chi_{L}$ are not sharp, and the signal is unclear. This may come from the small spatial and temporal sizes. In fact, the WHOT collaboration reported in a finite temperature simulation with the same action that the hopping parameter dependence of the Polyakov loop susceptibility shows a pronounced peak in a $16^{3} \times 6$ lattice [26].

The Polyakov loop phase $\phi$ and its susceptibility $\chi_{\phi}$ 

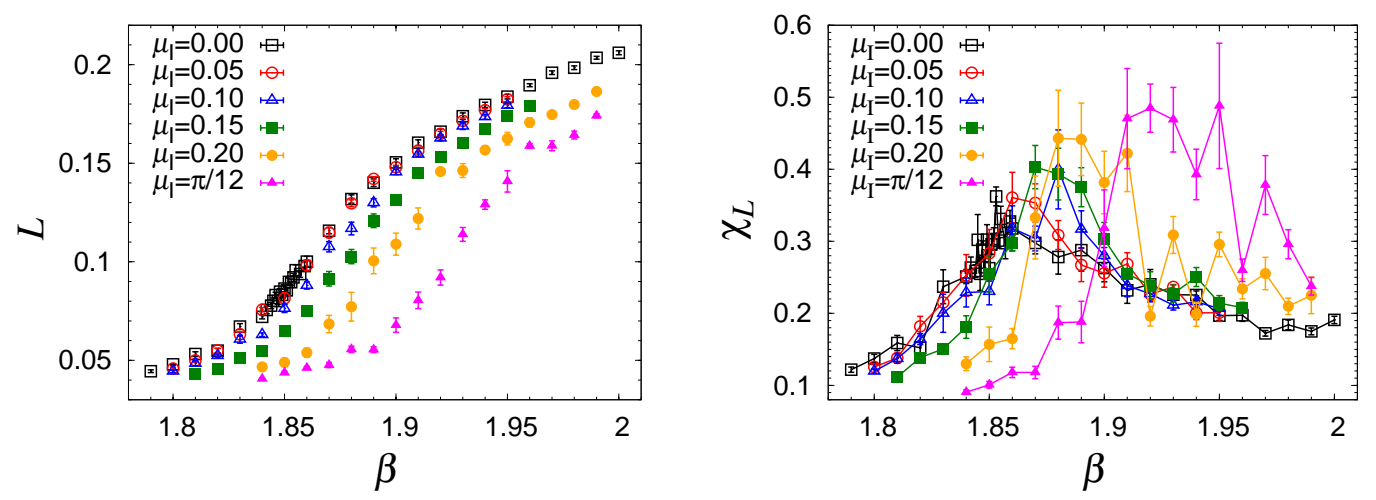

FIG. 5: The $\beta$-dependence of the Polyakov loop modulus $L$ (left) and its susceptibility $\chi_{L}$ (right) for various $\mu_{I}$.
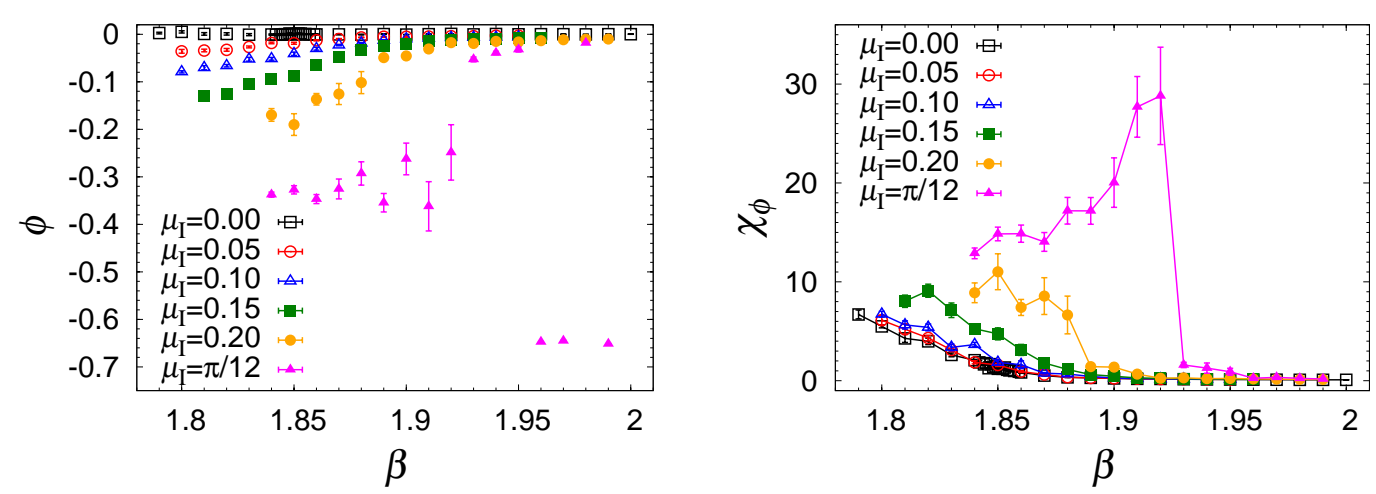

FIG. 6: The $\beta$-dependence of the Polyakov loop phase $\phi$ (left) and its susceptibility $\chi_{\phi}$ (right) for various $\mu_{I}$.

are shown in Fig. 6 $\phi$ rapidly changes near $\beta=1.92$ only for $\mu_{I}=\pi / 12$, while it is a smooth function of $\beta$ for $0 \leq \mu_{I}<\pi / 12$. It is seen that for $\mu_{I}=\pi / 12$ there is one vacuum at low temperatures and are two vacua at high temperatures. The histogram of $\phi$ at $\mu_{I}=\pi / 12$ in Fig. 7 also shows this behavior. The susceptibility $\chi_{\phi}$ shows a divergent-like behavior near $\beta=1.92$ only for $\mu_{I}=\pi / 12$. These behaviors suggest the possibility that the system undergoes the second order phase transition at the RW endpoint with increasing temperature.

The transition point of $\chi_{\phi}$ in Fig. 6 agrees with the the peak position of $\chi_{L}$ in Fig. 5 within error bars. Hence, the pseudo-critical line is connected with the Roberge-Weiss phase transition line at the RW endpoint. This is the case shown in Fig. 1. We observed that near the RW endpoint, the Polyakov loop modulus shows the crossover-like behavior and its phase shows the second order-like behavior. Note that the order of the phase transition were naively obtained from the behaviors of the observables. In order to confirm the order of the phase transition, the finite volume scaling analysis should be investigated in future works.

\section{B. RW phase transition}

Next, we show the $\mu_{I}$-dependence of the observables in Figs. 8 and 9 and investigate the nature of the RW phase transition line. Results of $P$ are not shown there, because the effect of $\mu_{I}$ changes the value of $P$ up to a few percent, as shown in the previous subsection.

$L$ decreases for $\mu_{I}<\pi / 12$, and increases for $\mu_{I}>$ $\pi / 12$. A rapidly decreasing behavior, which occurs due to the intersection with the pseudo-critical line, is ob- 


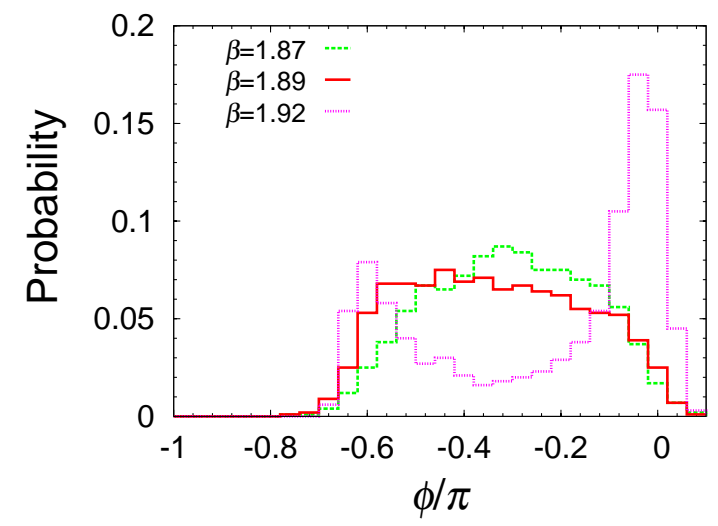

FIG. 7: The histogram of $\phi$ at $\mu_{I}=\pi / 12$ for various $\beta$.

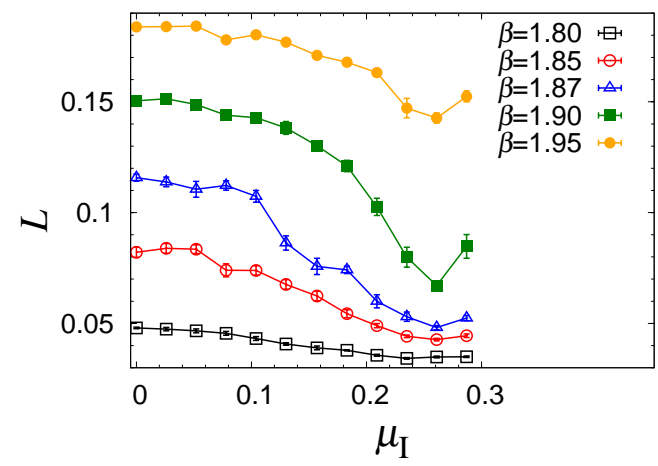

FIG. 8: The $\mu_{I}$-dependence of $L$ for various $\beta$.

served for $\beta=1.87$ and 1.90. The line for $\beta=1.95$ crosses the RW phase transition line at $\mu_{I}=\pi / 12$. However, a critical behavior is not observed there.

The $\mu_{I}$-dependence of $\phi$ and $\chi_{\phi}$ are shown in Fig. 9 $\phi$ is a smooth function of $\mu_{I}$ at low temperatures $(\beta=$ 1.80-1.90), while $\phi$ jumps to $-2 \pi / 3$ from 0 at $\mu_{I}=$ $\pi / 12$ at a high temperature $(\beta=1.95)$. The system undergoes the first order phase transition at $\mu_{I}=\pi / 12$ at high temperatures. Note $L$ is periodic and $\phi$ is antiperiodic, which is caused by the periodicity of the $\mu_{I^{-}}$ dependence of the Polyakov loop [6].

Together with the result obtained in the previous subsection, we find that the RW phase transition is the first order one and ends at second order endpoint, which are identified by the phase of the Polyakov loop. This feature is consistent with the results obtained in Ref. [7, 17]. It was reported [8, 10] that the order of the RW endpoint depends on the quark mass, and first order for light and heavy quark masses and second order for intermediate quark masses. Hence the second order nature of the RW endpoint comes from the intermediated quark mass.

\section{Pseudo-critical line}

As we have discussed in Sec. IIIA the Polyakov loop modulus $L$ shows the deconfinement crossover with increasing temperature, which spans the range $0 \leq \mu_{I} \leq \pi / 12$. We extract the value of $\beta_{p c}$ by fitting five or six data of $\chi_{L}$ near the peak with a Gaussian function: $\chi_{L} \propto \exp \left(-b\left(\beta-\beta_{p c}\right)^{2}\right)$.

TABLE I: The values of $\beta_{p c}$ determined by fitting $\chi_{L}$ with a Gaussian function.

\begin{tabular}{lll}
\hline$\mu_{I}$ & $\beta_{p c}$ & $\delta \beta_{p c}$ \\
\hline 0.00 & 1.866 & 0.007 \\
0.05 & 1.866 & 0.001 \\
0.10 & 1.877 & 0.008 \\
0.15 & 1.880 & 0.002 \\
0.20 & 1.891 & 0.001 \\
0.26 & 1.927 & 0.005 \\
\hline
\end{tabular}

The results are shown in Table I, and plotted as a function of $\mu_{I}^{2}$ in Fig. 10. The data points in Fig. 10 clearly deviate from a linear function near $\mu_{I} \sim \pi / 12$, which implies the contributions of higher-order terms. The need of terms of order higher than $\mu^{2}$ in the functional form of the pseudo-critical line was first pointed out in [14-16]. Such a behavior has not been obtained from a study with the combination of plaquette gauge action and the standard Wilson fermion without the clover term [17]. Hence the clover-improved Wilson fermion and the RG-improved gauge action leads to the deviation from a linear dependence of the pseudocritical line on $\mu_{I}^{2}$. This finding is an advantage of the improved actions.

In general, the pseudo-critical line can be expanded in powers of $\mu_{I}$ :

$$
\beta_{p c}\left(\mu_{I}\right)=\sum_{n} c_{n}\left(\mu_{I}^{2}\right)^{n},
$$



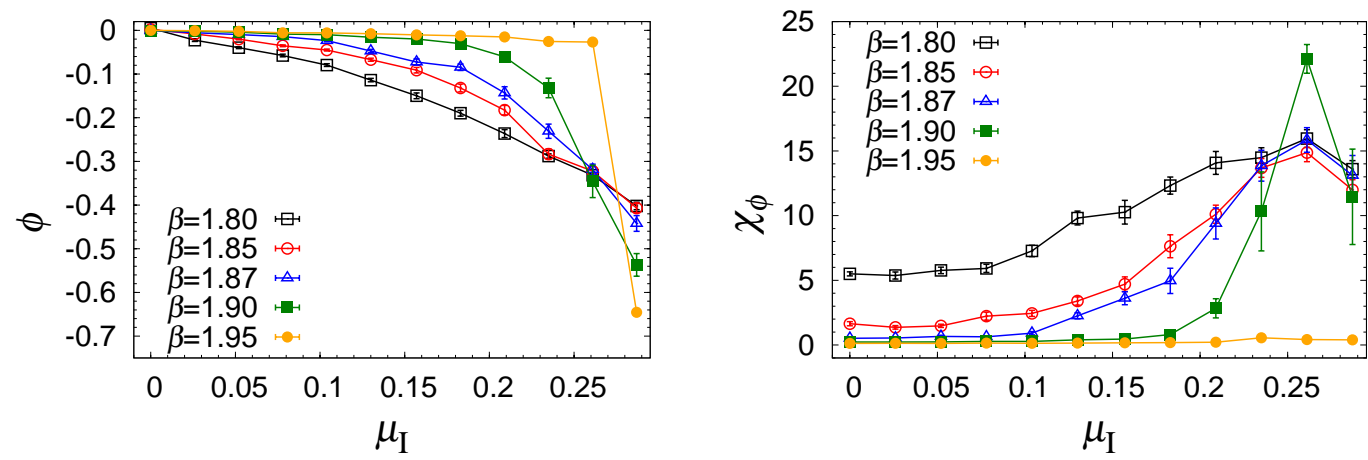

FIG. 9: The $\mu_{I}$-dependence of $\phi$ and $\chi_{\phi}$ for various $\beta$.

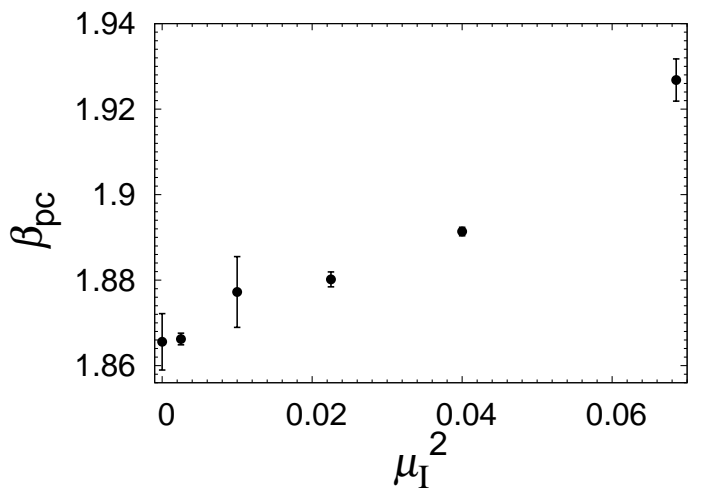

FIG. 10: $\beta_{c}$ and $\mu_{I}^{2}$.

which is defined for the range $0 \leq \mu_{I} \leq \pi / 12$ bounded by the RW endpoint. Within the definition range and with the present numerical results, only a few terms can be determined. Here, we test quadratic and quartic functions and a Padé approximation of a simple type

$$
\beta_{p c}\left(\mu_{I}\right)=c_{0} \frac{1+c_{1} \mu_{I}^{2}}{1+c_{2} \mu_{I}^{2}} .
$$

TABLE II: The coefficients of the fit functions for the pseudocritical line. $c_{0}$ is fixed with the central value of $\beta_{p c}(0)$.

\begin{tabular}{lcccc}
\hline type & $c_{0}$ & $c_{1}$ & $c_{2}$ & $\sqrt{\chi^{2} / \text { d.o.f }}$ \\
\hline quadratic & 1.866 & $0.67(4)$ & - & 1.56 \\
quartic & 1.866 & $0.40(9)$ & $6.64(2.17)$ & 0.96 \\
Padé & 1.866 & $-6.59(90)$ & $-6.84(88)$ & 0.76 \\
\hline
\end{tabular}

The results are shown in Table $\coprod$ and Fig. 11. The Padé approximation and quartic function are better than the quadratic function because of non- $\mu_{I}^{2}$ contributions, as we have mentioned above. The Padé approximation is slightly better than the quartic function in $\chi^{2} /$ d.o.f, but the difference is quite small in the $\mu^{2} \leq 0$ region. They are extended to the $\mu^{2} \geq 0$ plane through the analytic continuation $\mu_{I}^{2} \rightarrow-\mu^{2}$. The result in the $\mu^{2} \geq 0$ region depends on the fit functions in particular at large $\mu^{2}$.

The pseudo-critical line $\beta_{p c}\left(\mu_{I}\right)$ can be transformed into the one in physical unit through the relation

$$
T=\frac{1}{a(\beta) N_{t}} .
$$

However, we use the data for $\beta$ dependence of $T / T_{p c}(0)$ obtained in Ref. [26] instead of determining lattice spacings. Note that the value of $\beta_{p c}(0)$ slightly disagrees with the one obtained in Ref. [26], which causes the deviation of $T_{p c} / T_{p c}(0)$ at $\mu=0$ from 1 about $1 \%$

TABLE III: The coefficients of the fit functions for the pseudo-critical line.

\begin{tabular}{cccccc}
\hline type & $d_{0}$ & $d_{1}$ & $d_{2}$ & $d_{3}$ & $\sqrt{\chi^{2} / \text { d.o.f }}$ \\
\hline quadratic & 1.01 & $0.077(5)$ & - & - & 1.68 \\
quartic & 1.01 & $0.039(12)$ & $0.060(19)$ & - & 1.00 \\
Padé (I) & 1.01 & $-0.44(5)$ & $-0.49(5)$ & - & 0.70 \\
Padé (II) & 1.01 & $-0.89(10)$ & $-1.02(12)$ & $0.119(37)$ & 0.55 \\
\hline
\end{tabular}

It should be noted that $T$ is not a linear function of $\beta$, therefore there is no need that the above functions can 

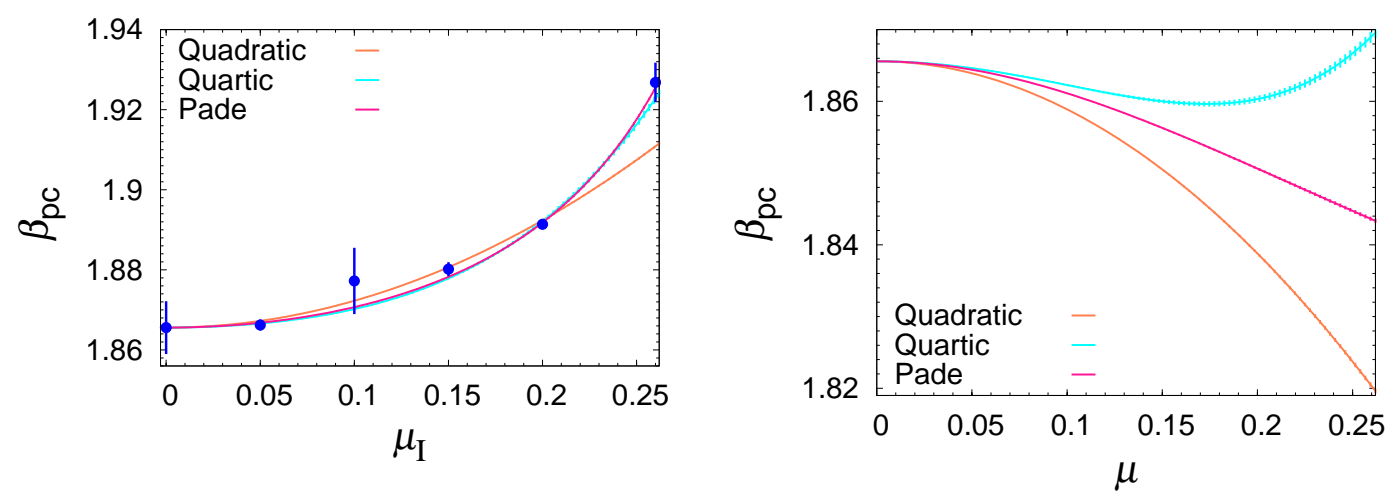

FIG. 11: The pseudo-critical line $\beta_{p c}$ in the imaginary(left panel) and real(right panel) region.
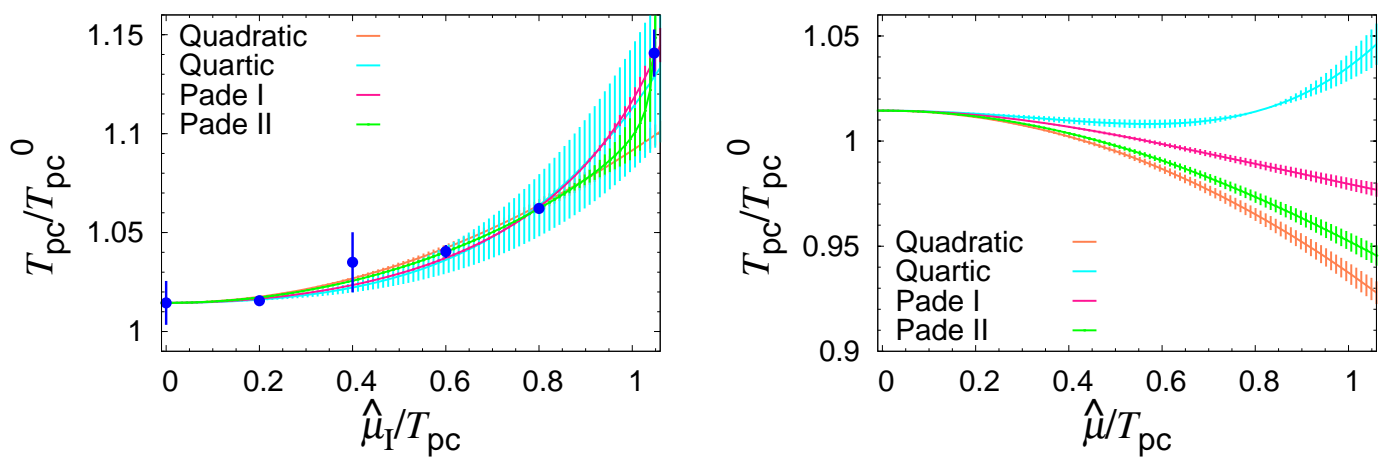

FIG. 12: The pseudo-critical line in dimensionless physical unit in the imaginary (left panel) and real(right panel) region.

be used here. We consider quadratic, quartic functions and two types of the Padé approximation.

$$
\begin{aligned}
\frac{T_{p c}}{T_{p c}^{0}} & =\sum_{n} d_{n}\left(\frac{\hat{\mu}_{I}}{T_{p c}}\right)^{2 n} \\
\frac{T_{p c}}{T_{p c}^{0}} & =d_{0} \frac{1+d_{1}\left(\hat{\mu}_{I} / T_{p c}\right)^{2}}{1+d_{2}\left(\hat{\mu}_{I} / T_{p c}\right)^{2}} \quad(\text { Padé }(\mathrm{I})), \\
\left(\frac{T_{p c}}{T_{p c}^{0}}\right)^{2} & =d_{0} \frac{1+d_{1}\left(\hat{\mu}_{I} / T_{p c}\right)^{2}}{1+d_{2}\left(\hat{\mu}_{I} / T_{p c}\right)^{2}+d_{3}\left(\hat{\mu}_{I} / T_{p c}\right)^{4}}
\end{aligned}
$$$$
\text { (Padé (II)), }
$$

where $\mu_{I}=a \hat{\mu}_{I}$, and $\hat{\mu}_{I}$ is the imaginary chemical potential in physical unit. $T_{p c}^{0}$ and $T_{p c}$ are pseudo-critical temperatures at zero and finite chemical potentials. As we have mentioned above, $d_{0}\left(=T_{p c} / T_{p c}(0)\right.$ at $\left.\mu=0\right)$ deviates from one with $1 \%$ because of the disagreement of $\beta_{p c}(0)$ from Ref. [26]. Here we added Padé (II) defined in Eq. (13), which was investigated to fit the critical line for four flavors in Ref. [14]. Note that Eq. (13) can be extended to $T_{p c}=0$ for the $\mu^{2}>0$ region [14], although the definition range of the line is restricted by the RW endpoint. The results are shown in Table III and Fig.12,

The central values of the quadratic, quartic and Padé (I) approximation show similar behavior to $\beta_{p c}\left(\mu_{I}\right)$. The Padé (II) are consistent with the quartic and Padé (I) until $\mu_{I} / T_{p c}<0.8$, and shows sharp rising for $0.8<\mu_{I} / T_{p c}$. Considering the errors, the quadratic function still undershoots the obtained data, and the quartic function suffers from large errors. The two Padé approximations reproduce the data well with small errors. The difference between the two Padé approximations are observed for $0.8<\mu_{I} / T$. Further investigations of this region would be important for better determination of the pseudo-critical line.

Similar to $\beta_{p c}$, the deviation becomes larger with increasing $\hat{\mu} / T$ in the $\hat{\mu}^{2} \geq 0$ region and amounts to 
more than $10 \%$ at $\hat{\mu} / T \sim 1$. The quartic function increases, and the other three functions decreases. The quartic function is completely different from the other three functions, although the quartic and Padé (I) are almost same in the $\mu^{2}<0$ region.

The quadratic function decreases the fastest, the Padé (II) does the next, and the Padé (I) overshoots the other two. The curvature at $\hat{\mu} / T_{p c}=0$ of a power series of $\left(\hat{\mu} / \pi T_{p c}\right)^{2}$ is often used to make comparisons with various studies [28]. We obtain

$$
t_{2}=\pi^{2} d_{1}=0.38(12)
$$

Here we employ the result for the quartic function. The result is smaller than the value obtained from staggered fermions [7, 29] and standard Wilson fermions [17] and implies the pseudo-critical line decreases slower.

\section{SUMMARY AND OUTLOOK}

We have investigated the two-flavor QCD phase diagram in the lattice QCD. The imaginary chemical potential approach was employed in order to avoid the sign problem. The clover-improved Wilson action and renormalization-group improved gauge action was first applied to the imaginary chemical potential approach. The simulation was performed on the $8^{3} \times 4$ lattice and at the intermediate quark mass. The simulation was performed for more than 150 points in the parameter $\left(\beta, \mu_{I}\right)$ plane. Considering the Polyakov loop, the imaginary chemical potential region of the phase diagram was examined.

Obtained behaviors of the phase transitions are first order for the RW phase transition, second order for the
RW endpoint and the crossover for the deconfinement. The corresponding order parameter for the phase transitions are the phase of the Polyakov loop for the RW phase transition and RW endpoint and the modulus of the Polyakov loop for the pseudo-critical line. The Polyakov loop modulus did not show a critical behavior on the RW phase transition line, while the Polyakov loop phase did not on the pseudo-critical line. We determined the pseudo-critical line from the susceptibility of the Polyakov loop modulus. We found a clear deviation from a linear dependence of the pseudo-critical line on $\mu_{I}^{2}$.

The present calculation was performed with the intermediate quark mass and small lattice. The finite volume scaling analysis and quark mass-dependence analysis are necessary to confirm the present results. In particular, the order of the RW endpoint depends on the mass of the quark. The improvement on these points should be done in a future study.

\section{Acknowledgment}

We would like to appreciate for Yuji Sakai, Kouji Kashiwa, Hiroaki Kouno, and Masanobu Yahiro for discussions and valuable comments. KN thanks the XQCD-J collaboration, Shinji Motoki, Yoshiyuki Nakagawa, and Takuya Saito for discussions.

The simulation was performed on NEC SX-8R at RCNP, and NEC SX-9 at CMC, Osaka University, and HITACHI SR11000 and IBM Blue Gene/L at KEK. This work was supported by Grants-in-Aid for Scientific Research 20340055 and 20105003.
[1] Z. Fodor, Proceedings of XL International Symposium on Multiparticle Dynamics, Univ. Antwerp, 2011 (2011), arXiv:.

[2] C. DeTar, (2011), arXiv:1101.0208

[3] S. Muroya, A. Nakamura, C. Nonaka, and T. Takaishi, Prog.Theor.Phys. 110, 615 (2003), arXiv:hep-lat/0306031

[4] P. de Forcrand, PoS LAT2009, 010 (2009), arXiv:1005.0539

[5] Y. Sakai, K. Kashiwa, H. Kouno, and M. Yahiro, Phys.
Rev. D77, 051901 (2008), arXiv:0801.0034

[6] H. Kouno, Y. Sakai, K. Kashiwa, and M. Yahiro, J. Phys. G36, 115010 (2009), arXiv:0904.0925.

[7] P. de Forcrand and O. Philipsen, Nucl. Phys. B642, 290 (2002), arXiv:hep-lat/0205016

[8] M. D'Elia and F. Sanfilippo, Phys. Rev. D80, 111501 (2009), arXiv:0909.0254

[9] M. D'Elia and F. Sanfilippo, Phys.Rev. D80, 014502 (2009), arXiv:0904.1400

[10] P. de Forcrand and O. Philipsen, Phys.Rev.Lett. 105, 
152001 (2010), arXiv:1004.3144

[11] M. D’Elia and M.-P. Lombardo, Phys.Rev. D67, 014505 (2003), arXiv:hep-lat/0209146.

[12] M. D'Elia and M. P. Lombardo, Phys.Rev. D70, 074509 (2004), arXiv:hep-lat/0406012.

[13] M. D’Elia, F. Di Renzo, and M. P. Lombardo, Phys.Rev. D76, 114509 (2007), arXiv:0705.3814

[14] P. Cea, L. Cosmai, M. D'Elia, and A. Papa, Phys.Rev. D81, 094502 (2010), arXiv:1004.0184

[15] P. Cea, L. Cosmai, M. D’Elia, C. Manneschi, and A. Papa, Phys.Rev. D80, 034501 (2009), arXiv:0905.1292

[16] P. Cea, L. Cosmai, M. D’Elia, and A. Papa, Phys.Rev. D77, 051501 (2008), arXiv:0712.3755.

[17] L.-K. Wu, X.-Q. Luo, and H.-S. Chen, Phys. Rev. D76, 034505 (2007), arXiv:hep-lat/0611035

[18] CP-PACS Collaboration, A. Ali Khan et al., Phys.Rev. D63, 034502 (2001), arXiv:hep-lat/0008011

[19] C. Bonati, G. Cossu, M. D'Elia, A. Di Giacomo, and C. Pica, PoS LATTICE2008, 204 (2008), arXiv:0901.3231

[20] C. W. Bernard et al., Phys.Rev. D49, 3574 (1994), arXiv:hep-lat/9310023

[21] Y. Iwasaki, K. Kanaya, S. Kaya, and T. Yoshie,
Phys.Rev.Lett. 78, 179 (1997), arXiv:hep-lat/9609022.

[22] A. Roberge and N. Weiss, Nucl. Phys. B275, 734 (1986).

[23] C.-N. Yang and T. Lee, Phys.Rev. 87, 404 (1952).

[24] T. Lee and C.-N. Yang, Phys.Rev. 87, 410 (1952).

[25] Y. Iwasaki, Nucl. Phys. B258, 141 (1985).

[26] WHOT-QCD Collaboration, S. Ejiri et al., Phys.Rev. D82, 014508 (2010), arXiv:0909.2121.

[27] B. Sheikholeslami and R. Wohlert, Nucl. Phys. B259, 572 (1985).

[28] O. Philipsen, Prog.Theor.Phys.Suppl. 174, 206 (2008), arXiv:0808.0672

[29] C. Allton et al., Phys.Rev. D66, 074507 (2002), arXiv:hep-lat/0204010.

[30] At zero and imaginary chemical potential a square-root is enough for studying $N_{f}=2$.

[31] Here we do not mean that a pseudo-critical line has an endpoint defined in statistical mechanics. Rather, we use a term endpoint for a situation where a change between the confinement and deconfinement phases becomes very continuous, and the pseudo-critical temperature is not well-defined. 